\title{
GENERALIZATION OF THE GROSS-PERRY METRICS
}

\author{
M. Jakimowicz and J. Tafel \\ Institute of Theoretical Physics, University of Warsaw, \\ Hoża 69, 00-681 Warsaw, Poland, email: tafel@fuw.edu.pl
}

\begin{abstract}
A class of $S O(n+1)$ symmetric solutions of the $(\mathrm{N}+\mathrm{n}+1)$-dimensional Einstein equations is found. It contains 5-dimensional metrics of Gross and Perry and Millward.
\end{abstract}

\section{Introduction}

An extra spatial dimension was indroduced by Kaluza and Klein (see e.g. [1]) in order to unify electromagnetism and gravity. Recently, due to the string theory, extra dimensions became a permanent part of the high energy theoretical physics. For instance, in braneworld models (see [2] for a review) matter fields are confined to a four-dimensional brane and gravity can propagate in higher dimensional bulk. These higher dimensional models motivate studying Einstein's equations in $D>4$ dimensions. Some techniques of the 4-dimensional Einstein theory were already generalized to higher dimensions. They refer mainly to the classification of the Weyl tensor, the Robinson-Trautman solutions , spacetimes with vanishing invariants and metrics with D-2 abelian symmetries (see e.g. [3, 4, 5, 6]).

Symmetry assumptions are one the most efficient methods of solving the Einstein equations. All well known multidimensional exact solutions like the Myers-Perry black hole [7, black ring of Emparan and Reall [8] and the Gross-Perry metrics [9] (see also [10]) admit several dimensional symmetry groups.

In [11] we proposed a construction of vacuum metrics admitting $S O(n+1)$ spherical symmetry, which was based on the symmetry reduction of $(N+n+1)$-dimensional Einstein equations to $(N+1)$-dimensional equations with a scalar field $\phi$. There was used an additional assumption that the field of normal vectors to surfaces $\phi=$ const is geodetic and the induced metric of the surfaces is an Einstein metric. The construction, for zero cosmological constant and timelike surfaces $\phi=$ const, can be summarized as follows.

Let $\gamma_{i j}$ and $P_{i j}$ be symmetric tensors depending on coordinates $x^{i}, i=0, \ldots, N-1$. Assume that $\gamma_{i j}$ has the Lorentz signature and $P_{i j}$ satisfies the following conditions

$$
\begin{gathered}
P_{i}^{i}=0, \\
P_{j}^{i} P_{i}^{j}=2 c=\text { const }, \\
P_{i ; k}^{k}=0
\end{gathered}
$$


where $P^{i}{ }_{j}=\gamma^{i k} P_{i j}$ and the semicolon denotes covariant derivative related to the metric $\gamma_{i j} d x^{i} d x^{j}$. From matrices $\gamma=\left(\gamma_{i j}\right)$ and $P=\left(P_{j}^{i}\right)$ we compose metric corresponding to $\gamma e^{P \tau}$, where $\tau$ is a function of another coordinate $s$. We assume that its Ricci tensor satisfies

$$
R^{i}{ }_{j}\left(\gamma e^{P \tau}\right)=\lambda \delta^{i}{ }_{j}, \quad \lambda=\text { const } .
$$

Given $c$ and $\lambda$ we look for solutions $\beta(s)$ and $\phi(s)$ of the following equations

$$
\begin{gathered}
(\beta \dot{\phi})^{\cdot}=-\beta V_{, \phi}, \\
-N \lambda \beta^{-2 / N}=\left(1-\frac{1}{N}\right) \frac{\dot{\beta}^{2}}{\beta^{2}}-\frac{2 c}{\beta^{2}}-\dot{\phi}^{2}+2 V,
\end{gathered}
$$

where the dot denotes the partial derivative with respect to $\mathrm{s}$ and $V$ is a function of $\phi$.

The main result of [11] is that, under conditions (11)-([6), metric

$$
\tilde{g}=-d s^{2}+\tilde{g}_{i j} d x^{i} d x^{j}
$$

were

$$
\tilde{g}_{i j}=\beta^{2 / N}\left(\gamma e^{P \tau}\right)_{i j}
$$

and $\tau(s)$ is defined via equation

$$
\beta \dot{\tau}=2
$$

satisfies $(N+1)$-dimensional Einstein equations with the scalar field $\phi$ and potential $V$. Moreover, if

$$
V=-\frac{1}{2} n(n-1) e^{-2 \sqrt{\frac{n+N-1}{n(N-1)}} \phi}
$$

then

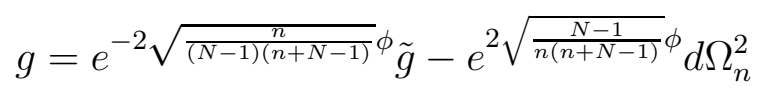

is an $(N+n+1)$-dimensional vacuum metric invariant under the group $S O(n+1)$. Here $d \Omega_{n}^{2}$ is the standard metric of the $n$-dimensional sphere.

A particular solution of conditions (11)-(4), for any $N>1$, is given by

$$
\gamma_{i j}=\operatorname{diag}(+1,-1,-1, . .), \quad P_{i j}=P_{j i}=\text { const }, \quad P_{i}^{i}=0, \lambda=0 .
$$

For $N=2$ conditions (11)-(4) can be solved in full generality. They lead either to (12) or to $c=0$ and to $\gamma e^{P \tau}$ equivalent to the metric

$$
\left(\gamma e^{P \tau}\right)_{i j} d x^{i} d x^{j}=\frac{d u d v}{\left(1+\frac{\lambda}{4} u v\right)^{2}}+\tau h(u) d u^{2}
$$

where $h$ is an arbitrary function of coordinate $u$. In the next section we find solutions of equations (5), (6) and construct corresponding vacuum metrics. In section 3 we discuss properties of these metrics. 


\section{Multi dimensional vacuum metrics}

In [11] we gave examples of vacuum metrics derived by our method. Other solutions with $n>1$ can be obtained by inspection of the Gross-Perry metrics [9]. Let $\lambda=0$ and $s=s(r)$ be a function of a new coordinate $r$. Then equations (5), (6) , (9) take the form

$$
\begin{gathered}
\left(\frac{\beta \phi^{\prime}}{\alpha}\right)^{\prime}=\alpha \beta V_{, \phi} \\
\left(1-\frac{1}{N}\right) \frac{\beta^{\prime 2}}{\alpha^{2}}-\frac{\beta^{2} \phi^{\prime 2}}{\alpha^{2}}+2 \beta^{2} V=2 c, \\
\tau^{\prime}=\frac{2 \alpha}{\beta},
\end{gathered}
$$

where the prime denotes the derivative with respect to $r$ and $\alpha=s^{\prime}$. Metric (7) is given by

$$
\tilde{g}=-\alpha^{2} d r^{2}+\beta^{2 / N}\left(\gamma e^{P \tau}\right)_{i j} d x^{i} d x^{j} .
$$

If $N=n=2$ equations (14), (15) are satisfied by functions $\alpha, \beta, \phi$ corresponding to the Gross-Perry metric [9]. Changing parameters in these functions leads to the following solutions for arbitrary dimensions $N>1$ and $n>1$

$$
\begin{gathered}
\alpha=\alpha_{0}|r|^{-l-1}\left|r-r_{0}\right|^{l-p}\left|r+r_{0}\right|^{l+p} \\
\beta=\beta_{0}\left(r^{2}-r_{0}^{2}\right) \alpha \\
e^{\sqrt{\frac{n+N-1}{n(N-1)}} \phi}=(n-1)|r \alpha| .
\end{gathered}
$$

Here $l$ is a number defined by $n$ and $N$

$$
l=\frac{n+N-1}{(n-1)(N-1)}
$$

and $p, \alpha_{0}, \beta_{0}$ and $r_{0} \neq 0$ are parameters related to the constant $c$ via

$$
c=2 \beta_{0}^{2} r_{0}^{2}\left[\frac{n}{n-1}-p^{2} \frac{(n-1)(N-1)^{2}}{N(n+N-1)}\right] .
$$

Integrating equation (16) yields

$$
\tau=\frac{1}{\beta_{0} r_{0}} \ln \left|\frac{r+r_{0}}{r-r_{0}}\right|+\tau_{0} .
$$

Due to a freedom of transformations of $r, P$ and $\gamma$ we can assume

$$
r_{0}>0, \quad\left|\alpha_{0}\right|=\frac{1}{n-1}, \quad \beta_{0}=1, \quad \tau_{0}=0
$$

(note that a sign of $\alpha_{0}$ can be still adjusted to have $\beta>0$ for $r \neq 0, \pm r_{0}$ ). Thus, $p$ and $r_{0}>0$ remain as free parameters. 
Let $N=2$. In the case (12) and $c>0$ the matrix $P$ can be diagonalized by a 2-dimensional Lorentz transformation. Hence, one obtains

$$
\tilde{g}=-\alpha^{2} d r^{2}+\beta\left(e^{ \pm \tau \sqrt{c}} d t^{2}-e^{\mp \tau \sqrt{c}} d y^{2}\right)
$$

where $t$ and $y$ denote coordinates $x^{i}$. Substituting (18)-(24) into (25) and (11) yields the following $(\mathrm{n}+3)$-dimensional vacuum metric

$$
g=\left|\frac{r-r_{0}}{r+r_{0}}\right|^{p^{\prime}-q} d t^{2}-\left|\frac{r-r_{0}}{r+r_{0}}\right|^{p^{\prime}+q} d y^{2}-\frac{\left|r+r_{0}\right|^{\frac{2 p^{\prime}+2}{n-1}}}{|r|^{\frac{2 n}{n-1}}\left|r-r_{0}\right|^{\frac{2 p^{\prime}-2}{n-1}}}\left(\frac{d r^{2}}{(n-1)^{2}}+r^{2} d \Omega_{n}^{2}\right)
$$

Parameters $p^{\prime}$ and $q$ are related to $p$ and $c$ by

$$
p^{\prime}=\frac{n-1}{n+1} p, \quad q= \pm \frac{\sqrt{|c|}}{r_{0}} .
$$

Because of (22) they are constrained by

$$
(n+1) p^{2}+(n-1) q^{2}=2 n .
$$

For $n=2$ solution (26) is exactly the Gross-Perry metric [9] under the identification

$$
r_{0}=m, \quad p^{\prime}=\frac{1}{\alpha}(\beta+1), \quad q=\frac{1}{\alpha}(\beta-1) .
$$

Here $m, \alpha$ and $\beta$ are parameters used by Gross and Perry, constrained by the condition $\alpha=\sqrt{\beta^{2}+\beta+1}$.

If $c<0$ the matrix $P$ can be put into the off diagonal form. Instead of (25) one obtains

$$
\tilde{g}=-\alpha^{2} d r^{2}+\beta\left[\cos (\tau \sqrt{|c|})\left(d t^{2}-d y^{2}\right) \pm 2 \sin (\tau \sqrt{|c|}) d t d y\right]
$$

In this case the vacuum metric corresponding to (18)-(24) reads

$$
\begin{aligned}
g= & \left|\frac{r-r_{0}}{r+r_{0}}\right|^{p^{\prime}}\left[\cos \left(q \ln \left|\frac{r+r_{0}}{r-r_{0}}\right|\right)\left(d t^{2}-d y^{2}\right)+2 \sin \left(q \ln \left|\frac{r+r_{0}}{r-r_{0}}\right|\right) d t d y\right] \\
& -\frac{\left|r+r_{0}\right|^{\frac{2 p^{\prime}+2}{n-1}}}{|r|^{\frac{2 n}{n-1}}\left|r-r_{0}\right|^{\frac{2 p^{\prime}-2}{n-1}}}\left(\frac{d r^{2}}{(n-1)^{2}}+r^{2} d \Omega_{n}^{2}\right) .
\end{aligned}
$$

Relation (27) is still valid, but now parameters $p^{\prime}, q$ are constrained by

$$
(n+1) p^{2}-(n-1) q^{2}=2 n .
$$

If $N=2$ and

$$
p= \pm \frac{\sqrt{2 n(n+1)}}{n-1}
$$


then it follows from (22) that $c=0$ and one can merge solutions (18)-(20) with metric (13) for $\lambda=0$. In this way the following vacuum metric is obtained

$$
\begin{aligned}
g= & \left|\frac{r-r_{0}}{r+r_{0}}\right|^{ \pm \sqrt{\frac{2 n}{n+1}}}\left(d u d v+\ln \left|\frac{r+r_{0}}{r-r_{0}}\right| h(u) d u^{2}\right) \\
& -\frac{\left|r+r_{0}\right|^{\frac{2}{n-1}}\left( \pm \sqrt{\frac{2 n}{n+1}}+1\right)}{|r|^{\frac{2 n}{n-1}}\left|r-r_{0}\right|^{\frac{2}{n-1}\left( \pm \sqrt{\frac{2 n}{n+1}}-1\right)}}\left(\frac{d r^{2}}{(n-1)^{2}}+r^{2} d \Omega_{n}^{2}\right)
\end{aligned}
$$

In the case $n=2, h(u)=0$ metric (34) with the lower sign coincides with the metric given by Millward [12] under the identification

$$
b=\frac{1}{\sqrt{3}} \ln \left|\frac{r-r_{0}}{r+r_{0}}\right|, M=\frac{\sqrt{3}}{2} r_{0} .
$$

For $N>2$ one can easily construct vacuum solutions based on relations (11), (12), (17)-(24). They generalize metrics (26) and (31). In this case a classification of symmetric tensors (here $P_{i j}$ ) in multidimensional Lorentzian manifolds [3] can be useful in order to distinguish nonequivalent solutions. One can also construct metrics which generalize (34) by taking $\gamma e^{P \tau}$ corresponding to the metric

$$
d u d v+\tau h(u) d u^{2}+\sum_{a=1}^{N-2} e^{c_{a} \tau} d y_{a}^{2},
$$

where constants $c_{a}$ are constrained by

$$
\sum_{a=1}^{N-2} c_{a}=0
$$

In this case we can use functions defined by (18)-(24) with constant $c$ given by

$$
c=\frac{1}{2} \sum_{a=1}^{N-2} c_{a}^{2} .
$$

\section{Discussion}

In adition to $S O(n+1)$ symmetries metrics (26) and (31) admit one timelike and one spacelike Killing vector (note that interpretation of $\partial_{t}$ and $\partial_{y}$ in case (31) can change depending on value of $r$ ). Metric (26) is static and metric (31) is stationary. In the limit $r \rightarrow \infty$ they behave like

$$
d t^{2}-d y^{2}-r^{-2 \frac{n-2}{n-1}}\left(\frac{d r^{2}}{(n-1)^{2}}+r^{2} d \Omega_{n}^{2}\right) .
$$


Under the change $r^{\prime}=r^{\frac{1}{n-1}}$ metric (39) takes the standard form of the $(\mathrm{n}+3)$-dimensional Minkowski metric. Thus, metrics (26) and (31) are asymptotically flat on surfaces $y=$ const.

Metric (34) has a null Killing vector field $\partial_{v}$ and it belongs to generalized Kundt's class [14]. If $r \rightarrow \infty$ it tends asymptotically to the flat metric in the form

$$
d u d v-r^{-2 \frac{n-2}{n-1}}\left(\frac{d r^{2}}{(n-1)^{2}}+r^{2} d \Omega_{n}^{2}\right) .
$$

Generalizing results of [13] for the Gross-Perry metric to arbitrary n, one can show that both metrics (26) and (31) are of algebraic type $I$. For $h \neq 0$ metric (34) is of algebraic type $I I_{i}$ and for $h(u)=0$ it is of type D. Aligned null vector fields for metrics (26), (31) and (34) are given in Appendix A.

All metrics 26), (31) and (34) are singular at $r= \pm r_{0}$ and $r=0$. Near $r=0$ they behave as

$$
d t^{2}-d y^{2}-r^{-\frac{2 n}{n-1}}\left(\frac{d r^{2}}{(n-1)^{2}}+r^{2} d \Omega_{n}^{2}\right) .
$$

Substituting $r^{\prime}=r^{-\frac{1}{n-1}}$ shows that (41) is the flat metric. Thus, $r=0$ is a coordinate singularity. By calculating the Kretschmann invariant (see Appendix B) it can be shown, that singularity at $r= \pm r_{0}$ is essential for all values of parameters in the case of metrics (26) and (34). In the case of metric (31) the singularity at $r=r_{0}$ and $r=-r_{0}$ is essential when, respectively, $p^{\prime}<n$ or $p^{\prime}>-n$. For $p^{\prime}>n$ or $p^{\prime}<-n$ the geodesic distance along $\partial_{r}$ tends to infinity when $r \rightarrow r_{0}$ or $r \rightarrow-r_{0}$, respectively. Thus, these regions represents an infinity different from that given by $r \rightarrow \infty$. For these values of parameters the Riemann tensor (in an orthonormal basis) tends to zero when $r \rightarrow r_{0}$ or $r \rightarrow-r_{0}$, respectively. However, the asymptotic metric is not the $(\mathrm{n}+3)$-dimensional Minkowski metric. Its coefficients in front of $d t$ and $d y$ tend to zero whereas the coefficient in front of $d \Omega_{n}^{2}$ tends to infinity like the geodesic distance to the power $2\left(p^{\prime}-1\right) /\left(p^{\prime}-n\right)$ or $2\left(p^{\prime}+1\right) /\left(p^{\prime}+n\right)$, respectively.

Since metrics (26), (31) are invariant under the nonnull field $\partial_{u}$ they can be interpreted in the context of the Kaluza-Klein theory. Then metric (26) is equivalent to the scalar field given by $g_{y y}$ and the asymptotically flat $(\mathrm{n}+2)$-dimensional metric induced on the surface $\mathrm{y}=$ const. The case $n=2$ (the Gross-Perry metric) was studied in this framework by Ponce de Leon [15]. In order to interpret metric (31) with $q \neq 0$ in this vein one can write it in the form

$$
g=-\Phi\left(d y-A_{0} d t\right)^{2}+g_{n+2} .
$$

Here

$$
A_{0}=\tan \left(q \ln \left|\frac{r+r_{0}}{r-r_{0}}\right|\right)
$$

is the electromagnetic potential,

$$
\Phi=\left|\frac{r-r_{0}}{r+r_{0}}\right|^{p^{\prime}} \cos \left(q \ln \left|\frac{r+r_{0}}{r-r_{0}}\right|\right)
$$


corresponds to a scalar field and

$$
g_{n+2}=\left|\frac{r-r_{0}}{r+r_{0}}\right|^{p^{\prime}} \frac{d t^{2}}{\cos \left(q \ln \left|\frac{r+r_{0}}{r-r_{0}}\right|\right)}-\frac{\left|r+r_{0}\right|^{\frac{2 p^{\prime}+2}{n-1}}}{|r|^{\frac{2 n}{n-1}}\left|r-r_{0}\right|^{\frac{2 p^{\prime}-2}{n-1}}}\left(\frac{d r^{2}}{(n-1)^{2}}+r^{2} d \Omega_{n}^{2}\right)
$$

defines, modulo a power of $\Phi$, a $(\mathrm{n}+2)$-dimensional metric. This metric is Lorentzian and asymptotically flat for large values of $r$ and becomes singular when $r$ diminishes to a value satisfying condition $q \ln \left|\frac{r+r_{0}}{r-r_{0}}\right|= \pm \pi / 2$.

Acknowledgements. This work was partially supported by the Polish Committee for Scientific Research (grant 1 PO3B 075 29).

\section{Appendix A}

The aligned null direction is given by

$$
\begin{aligned}
\hat{l} & =\left(\left|\frac{r-r_{0}}{r+r_{0}}\right|^{p^{\prime}-q}+\frac{1}{2} f^{2}\right) d t+\left(\left|\frac{r-r_{0}}{r+r_{0}}\right|^{p^{\prime}}-\frac{1}{2} f^{2}\left|\frac{r-r_{0}}{r+r_{0}}\right|^{q}\right) d y+ \\
& +f \frac{\left|r+r_{0}\right|^{\frac{p^{\prime}+1}{n-1}}}{(n-1)|r|^{\frac{n}{n-1}}\left|r-r_{0}\right|^{\frac{p^{\prime}-1}{n-1}}} d r
\end{aligned}
$$

for metric (26) and by

$$
\begin{aligned}
\hat{l} & =\left(\sin \left(q \ln \left|\frac{r+r_{0}}{r-r_{0}}\right|\right)+1\right)\left(1-\frac{1}{2} f^{2}\left|\frac{r-r_{0}}{r+r_{0}}\right|^{p^{\prime}} \frac{\cos \left(q \ln \left|\frac{r+r_{0}}{r-r_{0}}\right|\right)}{\left(\sin \left(q \ln \left|\frac{r+r_{0}}{r-r_{0}}\right|\right)+1\right)^{2}}\right) d t+ \\
& +\left(\frac{1}{2} f^{2}\left|\frac{r-r_{0}}{r+r_{0}}\right|^{p^{\prime}}-\cos \left(q \ln \left|\frac{r+r_{0}}{r-r_{0}}\right|\right)\right) d y+f \frac{\left|r+r_{0}\right|^{\frac{p^{\prime}+1}{n-1}}}{(n-1)|r|^{\frac{n}{n-1}}\left|r-r_{0}\right|^{\frac{p^{\prime}-1}{n-1}}} d r \quad \text { A.2 }
\end{aligned}
$$

for metric (31). The function $\mathrm{f}$ is a solution of the polynomial equation

$$
f^{4}-8 f^{2}\left(\frac{p^{\prime}}{q}+\frac{2 q(n-1) r r_{0}}{n\left(r^{2}+r_{0}^{2}-2 p^{\prime} r r_{0}\right)}\right) A-16 A^{2}=0
$$

where

$$
A=\left|\frac{r-r_{0}}{r+r_{0}}\right|^{p^{\prime}-q}
$$

in the case (26) and

$$
A=\left|\frac{r-r_{0}}{r+r_{0}}\right|^{-p^{\prime}}\left(\sin \left(q \ln \left|\frac{r+r_{0}}{r-r_{0}}\right|\right)+1\right)
$$


in the case (31). For metric (34) the aligned null directions are defined by

$$
\hat{n}=\left|\frac{r+r_{0}}{r-r_{0}}\right|^{ \pm \sqrt{\frac{2 n}{n+1}}} d u
$$

and

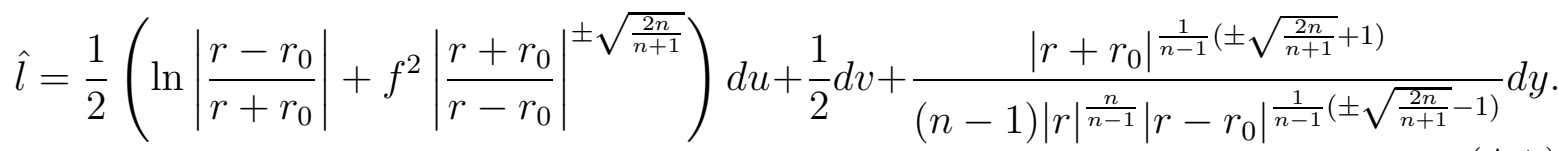

In this case

$$
f^{2}=\left(\mp \sqrt{\frac{n+1}{2 n}}+\frac{2\left(n^{2}-1\right) r r_{0}}{n(n+1)\left(r^{2}+r_{0}^{2}\right) \mp \sqrt{2 n(n+1)} r r_{0}}\right) h(u)\left|\frac{r+r_{0}}{r-r_{0}}\right|^{\mp \sqrt{\frac{2 n}{n+1}}} .
$$

\section{Appendix B}

The Kretschmann invariant for metrics (26) and (31) has the following form

$$
\begin{aligned}
R_{\mu \nu \delta \sigma} R^{\mu \nu \delta \sigma}= & 16 n(n-1) r_{0}^{2}|r|^{\frac{2(n+1)}{n-1}}\left|r-r_{0}\right|^{-\frac{4\left(n-p^{\prime}\right)}{n-1}}\left|r+r_{0}\right|^{-\frac{4\left(n+p^{\prime}\right)}{n-1}}\left(\left(n^{2}+n-2 p^{2}\right) r^{4}+\right. \\
& +2 p^{\prime}\left(n(n+1)\left(p^{2}-3\right)+2\left(1+p^{2}\right)\right) r^{3} r_{0}+ \\
& -\left(4-3 n-5 n^{2}-2(-2+n(n+3)) p^{\prime 2}+(4+n(n+3)) p^{\prime 4}\right) r^{2} r_{0}^{2}+ \\
& \left.+2 p^{\prime}\left(n(n+1)\left(p^{\prime 2}-3\right)+2\left(1+p^{\prime 2}\right)\right) r r_{0}^{3}+\left(n^{2}+n-2 p^{\prime 2}\right) r_{0}^{4}\right) .
\end{aligned}
$$

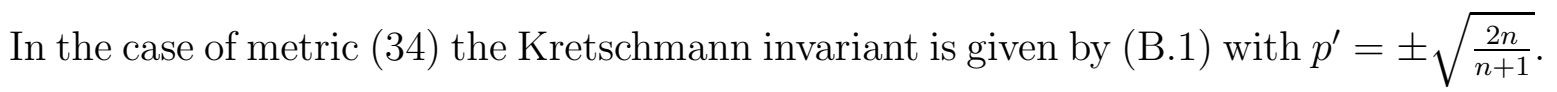

\section{References}

[1] Coquereaux R. and Jadczyk A. 1988, Riemannian geometry, fiber bundles, KaluzaKlein theories and all that..., World Scientific Lecture Notes in Physics 16

[2] Maartens R. 2004, Brane-world gravity, Living Rev. Relativity 7, (http://www.livingreviews.org/lrr-2004-7)

[3] Coley A., Milson R., Pravda V., Pravdova A. 2004, Classification of the Weyl Tensor in Higher Dimensions Class. Quantum Grav. 21 L35-L42

[4] Podolsky J. and Ortaggio M. 2006, Robinson-Trautman spacetimes in higher dimensions, Class. Quantum Grav., 2357855797

[5] Coley A., Fuster A., Hervik S., Pelavas N. 2006, Higher dimensional VSI spacetimes, Class. Quantum Grav., 23 7431-7444 
[6] Harmark T. 2004, Stationary and Axisymmetric Solutions of Higher-Dimensional General Relativity, Phys. Rev., D70 124002

[7] Myers R.C. and Perry M.J. 1986, Black holes in higher dimensional space-time, Annals Phys., 172304

[8] Emparan R. and Reall H.S. 2002, Rotating black ring in five dimensions, Phys. Rev. Lett., 88101101

[9] Gross D.J. and Perry M.J. 1983, Magnetic monopoles in Kaluza-Klein theories, Nucl. Phys., B 22629

[10] Davidson A. and Owen D. 1985, Black holes as a windows to extra dimensions Phys. Lett., B 155247

[11] Jakimowicz M. and Tafel J. 2008, SO(n + 1) symmetric solutions of the Einstein equations in higher dimensions, Class. Quantum Grav., 25175002

[12] Millward R.S. 2008, A five-dimensional Schwarzschild-like solution, arXiv: gr-qc/0603132

[13] Coley A. and Pelavas N. 2006, Classification of Higher Dimensional Spacetimes, Gen. Rel. Grav. 38 445-461

[14] Coley A. 2008, Classification of the Weyl Tensor in Higher Dimensions and Aplications, Class. Quantum Grav., 25033001

[15] Ponce de Leon J. 2007, Exterior spacetime for stellar models in 5-dimensional Kaluza-Klein gravity, Class. Quantum Grav. 241755 